ESAIM: PROCEEDINGS AND SURVEYS, November 2014, Vol. 46, p. 23-42

ECIT 2012 - Witold Jarczyk, Daniele Fournier-Prunaret, João Manuel Gonçalves Cabral

\title{
BIFURCATIONS AND CHAOS GENERATION FROM 1D OR 2D CIRCUITS INCLUDING SWITCHES
}

\author{
DaniÈle Fournier-Prunaret ${ }^{1}$, PASCAl Chargé $^{2}$ AND LAURA Gardini ${ }^{3}$
}

\begin{abstract}
Generation of chaos is of the highest interest for many kind of applications as secure transmissions, image processing or telecommunications. In order to obtain chaotic signals that can be used in applications, simple circuits including switches have been considered those last years. Their interest is of two types: first, they belong to the class of hybrid systems that have recently attracted a great interest, secondly, they permit to obtain chaos in a very easy way. Such circuits are very easy to implement and robust chaos can be obtained, depending upon parameter values. For this aim, it is necessary to study and understand the bifurcation structures of the circuit model. In this paper, we propose two kinds of chaos generators obtained from simple RC circuits including switches managed using a clock (impulse waveform) and the charging/discharging of the capacitors. Our models are given via one-dimensional (1D) or two-dimensional (2D) nonlinear maps. We present the bifurcation studies permitting to understand the evolution of the behaviour of our systems and the appearance of chaos. Keywords. chaotic signal; bifurcation; switching circuit; piecewise-smooth map.

AMS classification. $94 ; 37 \mathrm{Gxx} ; 65 \mathrm{Pxx}$.

Résumé. L'utilisation du chaos présente un grand intérêt dans beaucoup d'applications en lien avec les télécommunications, le traitement d'images ou les transmissions sécurisées. Des circuits très simples comprenant des commutations peuvent être utilisés pour produire des signaux chaotiques. Leur intérêt est double, tout d'abord ils appartiennent à la classe des systèmes hybrides qui ont récemment attiré l'attention, ensuite, ils permettent de générer des signaux chaotiques robustes de manière très simple et ils sont faciles à implémenter. Néanmoins il est nécessaire d'étudier et de comprendre les structures de bifurcations sous-jacentes. Dans cet article, nous proposons deux sortes de circuits RC très simples comportant des commutations qui utilisent une horloge afin de charger ou décharger les capacités. Les modèles proposés sont basés sur des récurrences de dimension un ou deux. Les structures de bifurcation des deux circuits sont étudiées et permettent de comprendre la génération de signaux chaotiques.
\end{abstract}

\section{INTRODUCTION}

The concept of chaos has been introduced during the second half of the twentieth century. Chaos corresponds to a kind of irregular behaviour, which can be obtained in nonlinear dynamical systems in many areas, especially in physical systems, for instance nonlinear circuits. Chua's circuit [18] or Duffing oscillator [11] are classical examples, more recently, MicroElectroMechanical Systems (MEMS) [3] have been studied in detail. The first researches about chaos have concerned its analysis, and more particularly the understanding of route to chaos by studying bifurcations giving rise to chaos. Then, during the two last decades, many researchers have tried to find

${ }^{1}$ LAAS, CNRS, INSA, Université de Toulouse, France, email: daniele.fournier@insa-toulouse.fr

2 IETR, CNRS, Polytech Nantes, University of Nantes, France, email: Pascal.Charge@univ-nantes.fr

${ }^{3}$ University Carlo Bo, Urbino, Italy, email: laura.gardini@uniurb.it

(C) EDP Sciences, SMAI 2014 
applications to chaotic signals, which also look like random signals. Some applications concern the use of chaotic signals for secure transmissions, telecommunications and image processing [19] [24]. It appears that all chaotic signals cannot be used in such applications. Indeed, even if chaotic signals look random, depending upon their origin, they do not have the same properties. Specific properties are required for specific applications, among them : i) broad spectrum, (ii) robustness of chaos to small parameter variations [1], (iii) specific bifurcations giving rise to chaos in an easy way. Good candidates with respect to these properties can be circuits including switches. Such systems are of interest for two reasons: first, they belong to the class of hybrid systems that have attracted much interest those last ten years, secondly, they can give rise to chaotic signals, which verify the properties (i)(ii)(iii). Chaotic generators can be obtained using continuous time models as systems based on the Chua's circuit (analogical circuit), while others are discrete time systems which directly iterate a chaotic map (digital circuit). The problem with these systems is that chaos is not necessarily as robust, in the sense given in [1]. Hybrid systems usually evolve in continuous time, but it is possible to model them using discrete time maps by introducing a discretization similar to the building of a Poincaré map. A way to obtain robust chaotic signals is to consider systems where border collision bifurcations appear [2] [16] [12] [13] [23], such systems constitute a class of hybrid systems. In this paper, we consider two kinds of hybrid circuits modeled using discrete time maps and we analyze their dynamics.

The chaos generators that we propose are obtained from simple RC circuits including switches managed using a clock (impulse waveform) and the charging/discharging of the capacitors. Their models are given via one-dimensional (1D) or two-dimensional (2D) nonlinear maps. Previous studies of such circuits have been done in [4] [6] [7] [8] [9] [10]. Our aim is to present the analogy between both kinds of circuits and the bifurcation studies. In this paper, we consider chaos and chaotic attractors in the sense given in [5], [22]. The section 2 is devoted to the description of the circuits and the recall of their modeling. Both kinds of models are based on the definition of piecewise continuous maps using three determinations. In section 3, bifurcation studies permitting to obtain periodic orbits and chaos are recalled.

\section{DESCRIPTION OF THE CIRCUITS}

We consider two simple RC circuits including switches, the first one is modeled via a one-dimensional (1D) map and the second one via a two-dimensional (2D) map. They both depend upon parameters that can vary and give rise to chaos. Both maps (1D and 2D) are piecewise continuous with three determinations. Now, we describe the two circuits and their modeling.

\subsection{The 1D circuit}

The 1D proposed chaos generator is a very simple circuit, which is sketched in Figure 1; it is very similar to those discussed in [16] [12]. The state variable is $v(t)$, the voltage across the capacitor. The switchings are given by a logical part including the clock, the two R-S latches and the logical functions $X O R$ and $A N D$. A switch occurs at every clock period $T$ or when the state variable $v(t)$ reaches the value of $V_{D}$ or $V_{U}$. The capacitor charges $(\mathrm{p}=0)$ when $v(t)$ reaches $V_{D}$ or discharges $(\mathrm{p}=1)$ when $v(t)$ reaches $V_{U}$. Then, we can use the classical equations of a RC circuit to obtain a model describing the possible states of the circuit. For more details on the functioning of the circuit and its modelization, see [9]. After normalization of the variables and the parameters in order to deal with dimensionless values and a normalized phase space equal to $[0,1]$, the circuit is modelled by the map $x_{n+1}=F\left(x_{n}\right)$ (cf. Figure 2), which is defined as follows:

$$
\begin{aligned}
& \text { if } x_{n} \in I_{1}=\left[0, x_{a}\left[, \quad F\left(x_{n}\right)=F_{1}\left(x_{n}\right)=1-\left(1-x_{n}\right) \delta\right.\right. \\
& \text { if } x_{n} \in I_{2}=\left[x_{a}, x_{c}\left[, \quad F\left(x_{n}\right)=F_{2}\left(x_{n}\right)=\left(1-x_{n}\right) \frac{\delta \beta}{1-\beta}\right.\right. \\
& \text { if } x_{n} \in I_{3}=\left[x_{c}, 1\right], \quad F\left(x_{n}\right)=F_{3}\left(x_{n}\right)=1-\left(1-x_{n}\right) \frac{\delta}{m} \frac{1-\beta m}{1-\beta}
\end{aligned}
$$




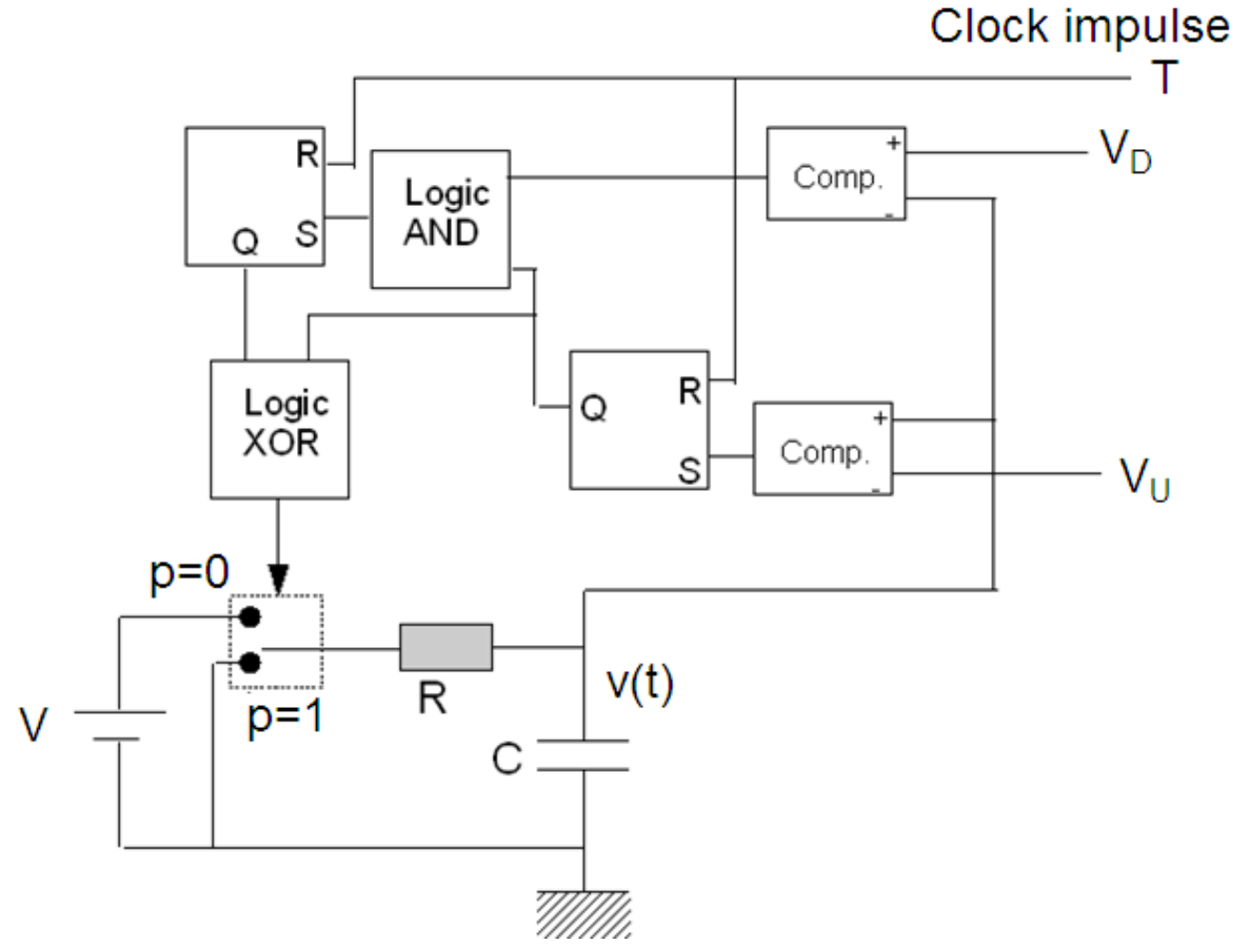

Figure 1. The RC 1D circuit with the logical part including the clock and the two R-S latches.

where the state variables and the parameters are:

$$
\begin{aligned}
& x_{n} \in[0,1], \quad x_{n+1} \in[0,1] \\
& \beta \in] 0,1[, \quad 0<m<1, \quad \delta \in] 0,1]
\end{aligned}
$$

with the switching values:

$$
x_{a}=1-\frac{1-\beta}{\delta}, \quad x_{c}=1-\frac{m(1-\beta)}{\delta}
$$

The map (1) is well defined as $F$ is continuous (piecewise linear) and $F$ maps the interval $[0,1]$ (the phase space of interest) into itself. $F$ is defined using the three different determinations $F_{1}, F_{2}$ and $F_{3}$ depending upon the location of the state variable in $[0,1]$.

\subsection{The $2 \mathrm{D}$ circuit}

The 2D circuit is shown in Figure 3 and its model has been introduced in a more detailed way in [4] [7] [10]. Just recall that the state variables of the system are the two voltage capacitors $v_{x}(t)$ and $v_{y}(t)$. At every clock period $T$, the flip-flop is set and then the switches position is ' 1 '. When one of the capacitance voltages reaches the reference value $V_{\text {ref }}$, the two switches are turned toward their position '0'. So, according to the switches position, the two capacitors are simultaneously charging or discharging. Thus, using classical models of circuits, we easily obtain the equations of the system. As in the $1 \mathrm{D}$ case, we normalize the state variables and the parameters. Then we obtain the 2D map $\left(x_{n+1}, y_{n+1}\right)=G\left(x_{n}, y_{n}\right)$ defined as follows in $Q=[0,1] \times[0,1]$ : 


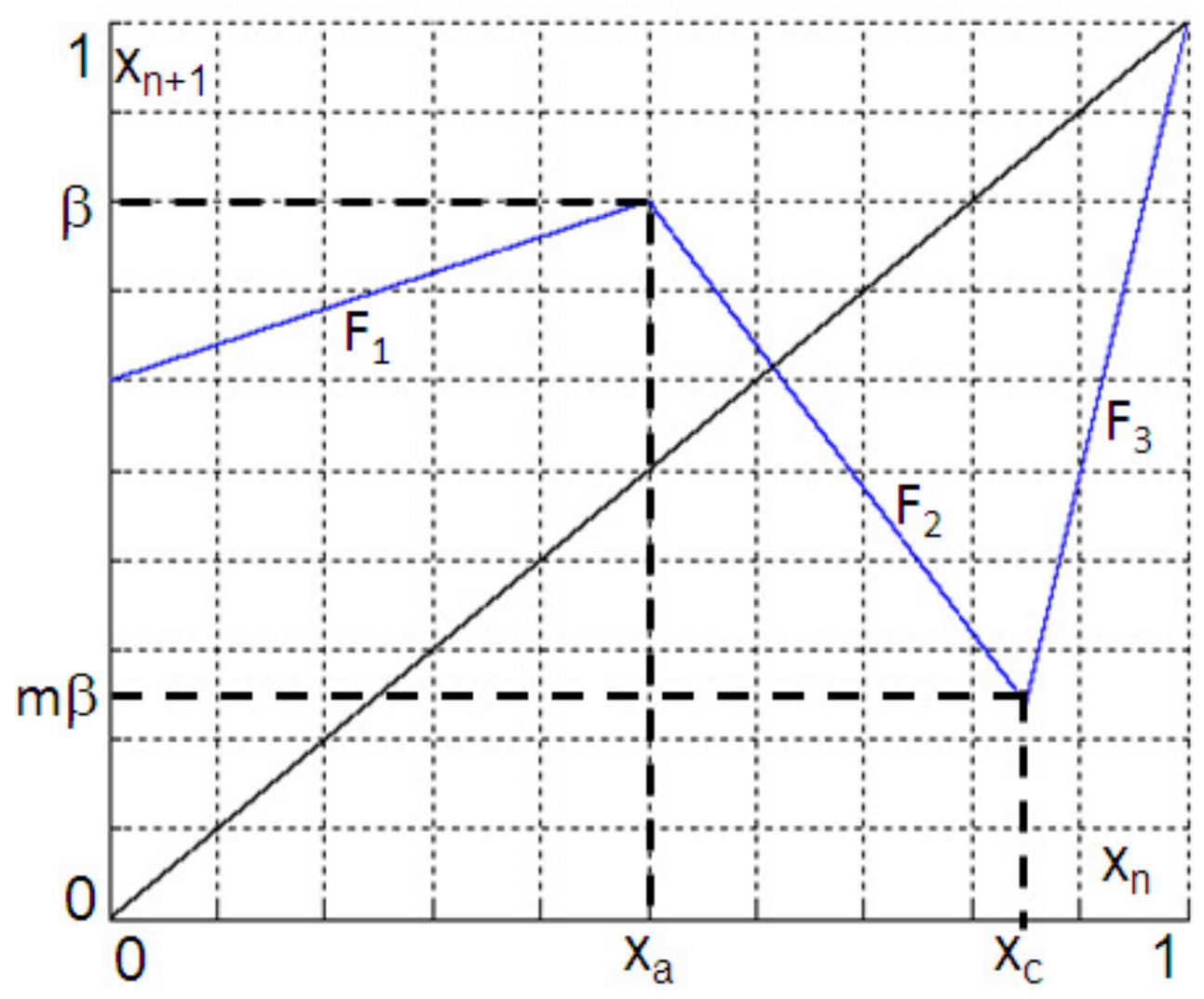

Figure 2. The 3-pieces piecewise linear map (1).

$$
\begin{aligned}
& \text { if }\left(x_{n}, y_{n}\right) \in D_{1}: \\
& G\left(x_{n}, y_{n}\right)=G_{1}\left(x_{n}, y_{n}\right)=\left\{\begin{array}{l}
\alpha+\left(x_{n}-\alpha\right) \delta \\
\alpha \rho+\left(y_{n}-\alpha \rho\right) \delta^{1 / \mu}
\end{array}\right. \\
& \text { if }\left(x_{n}, y_{n}\right) \in D_{2}: \\
& G\left(x_{n}, y_{n}\right)=G_{2}\left(x_{n}, y_{n}\right)=\left\{\begin{array}{l}
\frac{\alpha-x_{n}}{\alpha-1} \delta \\
\left(\alpha \rho\left(\frac{\alpha-x_{n}}{\alpha-1}\right)^{1 / \mu}-\alpha \rho+y_{n}\right) \delta^{1 / \mu}
\end{array}\right. \\
& \text { if }\left(x_{n}, y_{n}\right) \in D_{3}: \\
& G\left(x_{n}, y_{n}\right)=G_{3}\left(x_{n}, y_{n}\right)=\left\{\begin{array}{l}
\left(\alpha\left(\frac{\alpha \rho-y_{n}}{\alpha \rho-1}\right)^{\mu}-\alpha+x_{n}\right) \delta \\
\left(\frac{\alpha \rho-y_{n}}{\alpha \rho-1}\right) \delta^{1 / \mu}
\end{array}\right.
\end{aligned}
$$

with:

$$
\begin{aligned}
& x_{n} \in[0,1], \quad x_{n+1} \in[0,1] \\
& \alpha>1, \quad \rho \alpha>1, \quad \mu>0, \quad \delta \in] 0,1]
\end{aligned}
$$

where the three domains $D_{1}, D_{2}$ and $D_{3}$ are defined as follows (see Figure 4): 


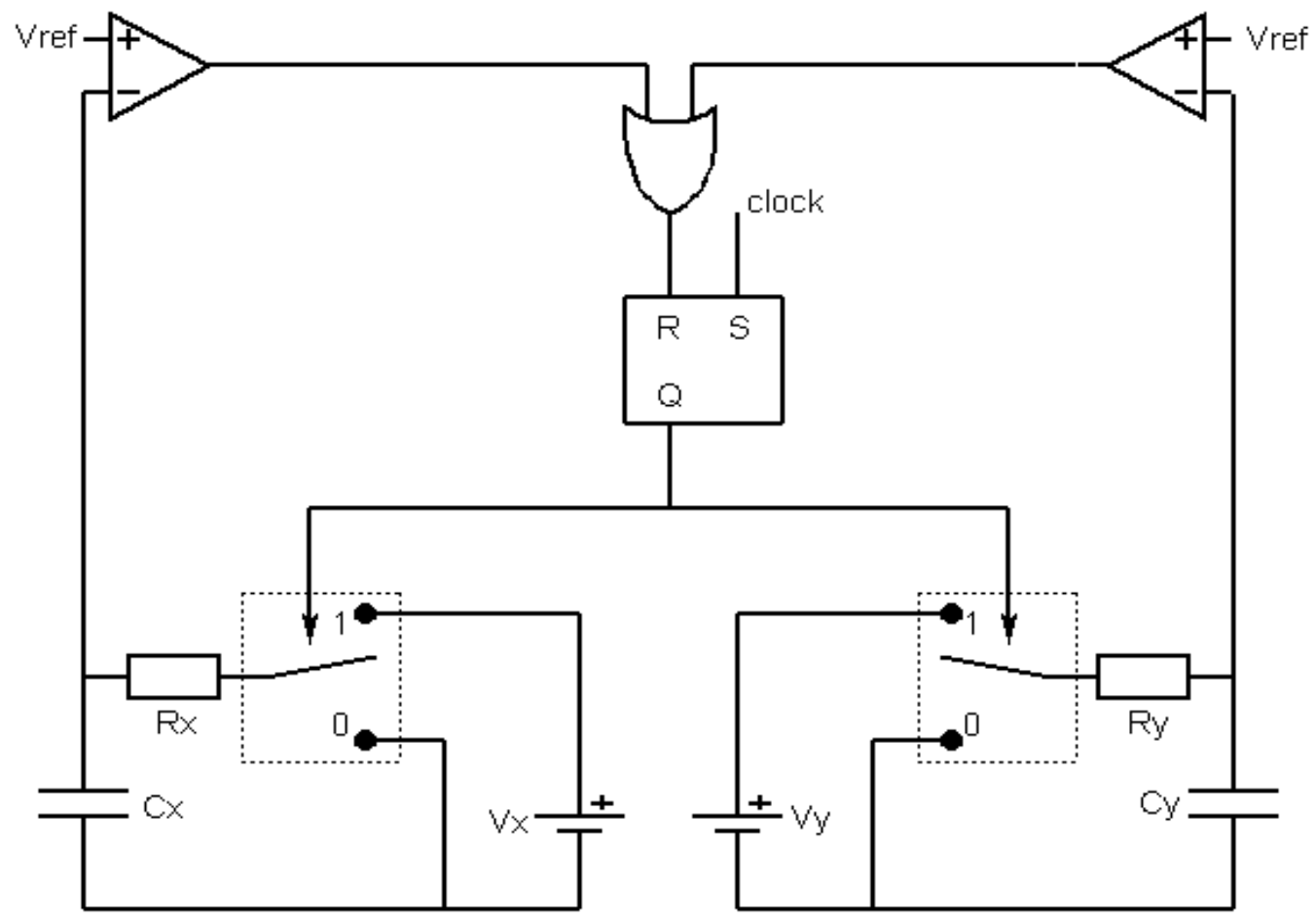

Figure 3 . The $2 \mathrm{D}$ circuit.

$$
\begin{aligned}
& D_{1}=\left\{(x, y) \mid 0 \leq x \leq x_{b} \text { and } 0 \leq y \leq y_{b}\right\} \\
& D_{2}=\left\{(x, y) \mid x_{b} \leq x \leq 1 \text { and } \Delta(x, y) \geq 0\right\} \\
& D_{3}=\left\{(x, y) \mid y_{b} \leq y \leq 1 \text { and } \Delta(x, y) \leq 0\right\}
\end{aligned}
$$

with the switching values in $Q=[0,1] \times[0,1]$ :

$$
\begin{aligned}
& x=x_{b} \quad, \quad x_{b}=\alpha-\frac{\alpha-1}{\delta} \\
& y=y_{b}, \quad y_{b}=\alpha \rho-\frac{\alpha \rho-1}{\delta^{1 / \mu}} \\
& \Delta(x, y)=0 \quad, \quad \Delta(x, y)=\left(\frac{\alpha \rho-y}{\alpha \rho-1}\right)^{\mu}-\frac{\alpha-x}{\alpha-1}
\end{aligned}
$$

assuming $x_{b} \geq 0$ and $y_{b} \geq 0$, which occurs for

$$
\bar{\delta}<\delta<1, \bar{\delta}=\max \left\{\frac{\alpha-1}{\alpha},\left(\frac{\alpha \rho-1}{\alpha \rho}\right)^{\mu}\right\}
$$

It is easy to see that the map is well defined as $G$ is continuous and maps the square $Q$ (the phase space of interest) into itself. As in the $1 \mathrm{D}$ case, the map is defined using three determinations $G_{1}, G_{2}$ and $G_{3}$, depending upon the location of the state variables in $Q$. 


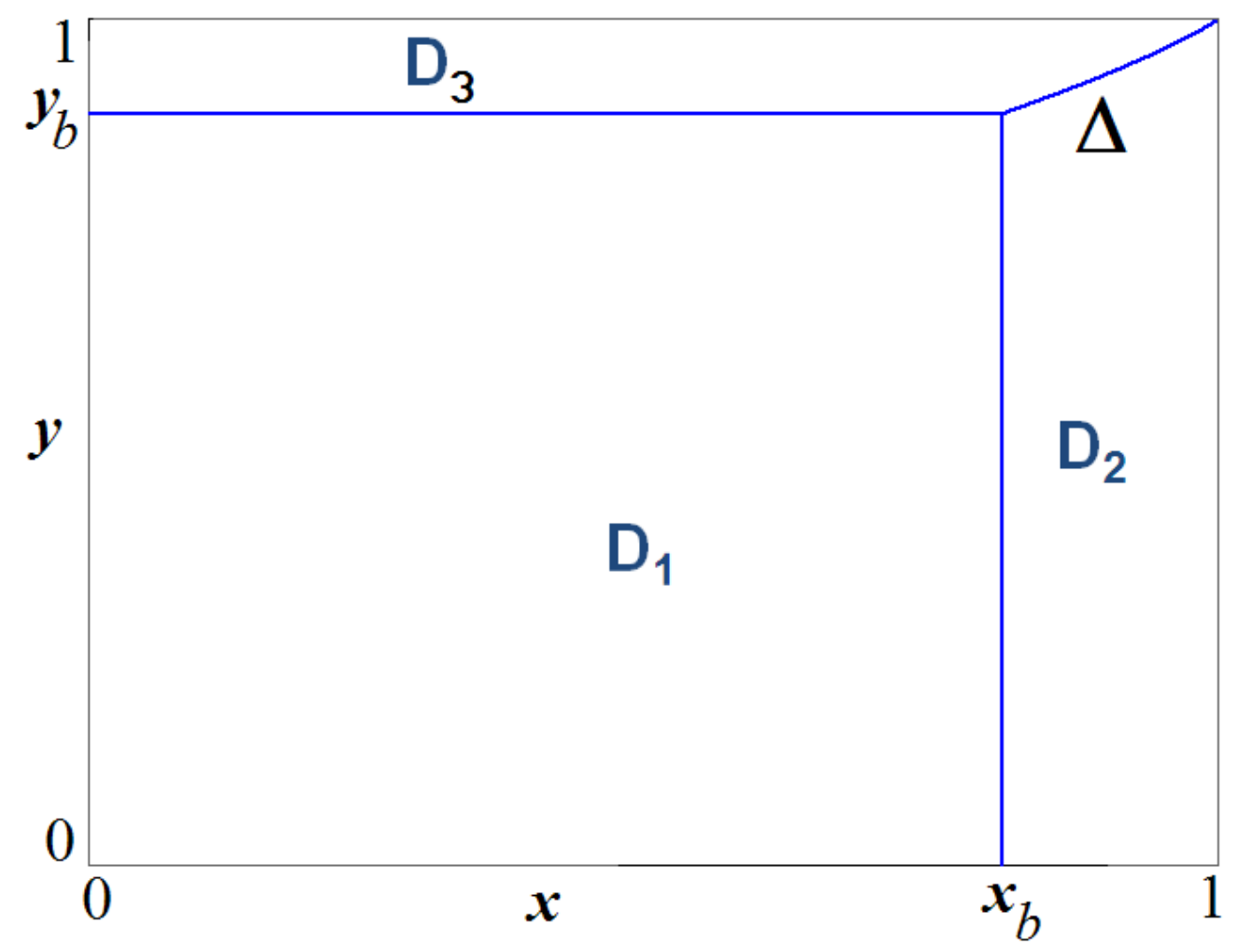

Figure 4. Phase space $Q$ of the map $G(4)$ and the three different regions $D_{i}, i=1,2,3$ where the three different determinations $G_{1}, G_{2}$ and $G_{3}$ are defined.

\section{Study OF BIFURCATIONS AND CHAOS IN THE TWO CIRCUITS}

\subsection{The 1D circuit}

The map (1) is a 1-dimensional piecewise linear map with three pieces. In this section, we recall results about the fixed points, order $k$ periodic orbits and their bifurcations [9]. We also put in evidence the homoclinic bifurcations giving rise to chaotic behaviour. First, let us give the slopes of the map, depending where is located the point in the interval $[0,1]$. These values will be useful for the rest of our study.

$$
\begin{aligned}
& \text { if } x \in\left[0, x_{a}\left[, \quad p_{1}=\delta>0\right.\right. \\
& \text { if } x \in\left[x_{a}, x_{b}\left[, \quad p_{2}=\frac{-\delta \beta}{1-\beta}<0\right.\right. \\
& \text { if } x \in\left[x_{b}, 1\right], \quad p_{3}=\frac{\delta}{m} \frac{1-\beta m}{1-\beta}>0
\end{aligned}
$$

\subsubsection{Fixed points}

We recall the results about fixed points, see for instance [8] or [9] for more details. Two fixed points $X_{1}^{*}$ and $X_{2}^{*}$ exist and are given by:

$$
\begin{aligned}
& X_{1}^{*}=\frac{\delta \beta}{1-\beta+\delta \beta} \in\left[x_{a}, x_{b}\right] \\
& X_{2}^{*}=1
\end{aligned}
$$


$X_{1}^{*}$ is stable when the slope $\left.p_{2}=\frac{-\delta \beta}{1-\beta} \in\right]-1,0\left[\right.$, that means $\beta<\frac{1}{1+\delta}$. $X_{2}^{*}$ is stable when the slope $\left.p_{3}=\frac{\delta}{m} \frac{1-\beta m}{1-\beta} \in\right] 0,1\left[\right.$, that means $\beta<\frac{m-\delta}{m(1-\delta)}$ and $m>\delta$. So, we can define the bifurcation curves related to fixed points bifurcations:

$$
\begin{aligned}
& F B 1 a: \beta=\frac{1}{1+\delta}, m<\delta, \quad F B 1 b: \beta=\frac{1}{1+\delta}, m>\delta \\
& F B 1 c: \beta=\frac{m-\delta}{m(1-\delta)}, m>\delta
\end{aligned}
$$

These curves correspond to degenerate flip bifurcations, FB1a corresponds to the appearance of a stable order 2 cycle after the flip bifurcation of $X_{1}^{*}, F B 1 b$ corresponds to the appearance of an order 2 cyclic chaotic attractor $\left(C_{1}, C_{2}\right)$, after $X_{1}^{*}$ has changed its stability; indeed, the order 2 periodic orbit $\left(Y_{1}, Y_{2}\right)$, which appears at the flip bifurcation $F B 1 b$, undergoes in the same time a border collision bifurcation $\left(Y_{2}\right.$ is merging with $\left.x_{b}\right)$ and becomes unstable (see Fig. 5). FB1c corresponds to a border collision bifurcation for $X_{1}^{*}$, which disappears when merging with $x_{b}$; at the same bifurcation value, $X_{2}^{*}$ becomes a stable fixed point. The curve $F B 1 c$ also corresponds to a degenerate bifurcation for $X_{2}^{*}$ with the appearance of an order 2 cyclic chaotic attractor, after $X_{2}^{*}$ has become unstable (see Fig. 6). Then, the order 2 cyclic chaotic attractor, after an homoclinic bifurcation, gives rise to an one-piece chaotic attractor (see Fig. 7). Such chaos can be considered as robust in the sense of remaining chaotic even if the parameters or the initial conditions are slightly changed [1].

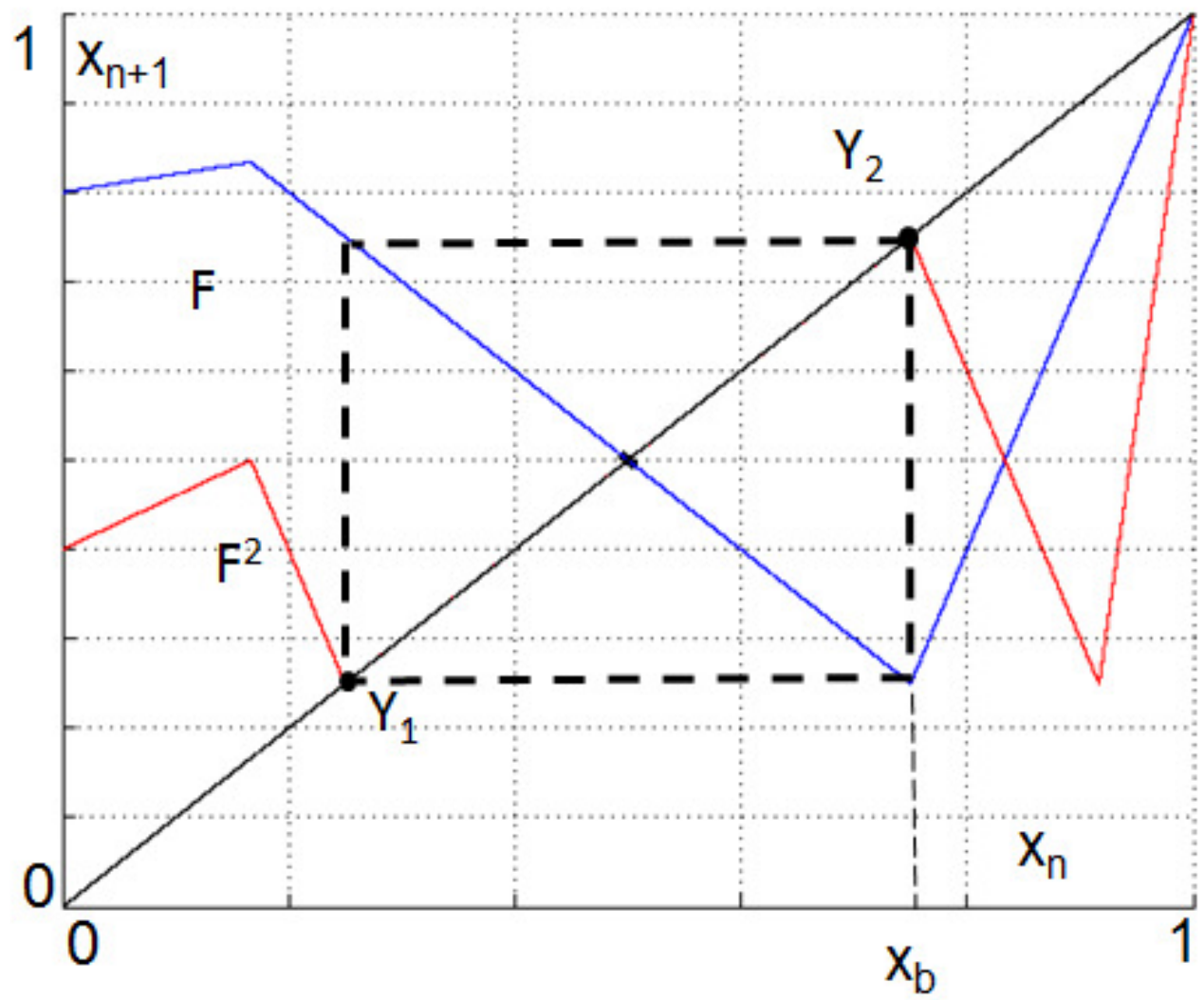

Figure 5. Map (1). Degenerate flip bifurcation $(F B 1 b)$ in the plane $\left(x_{n}, x_{n+1}\right)$ for $\delta=0.2$, $m=0.3$ and $\beta=0.8333$. 


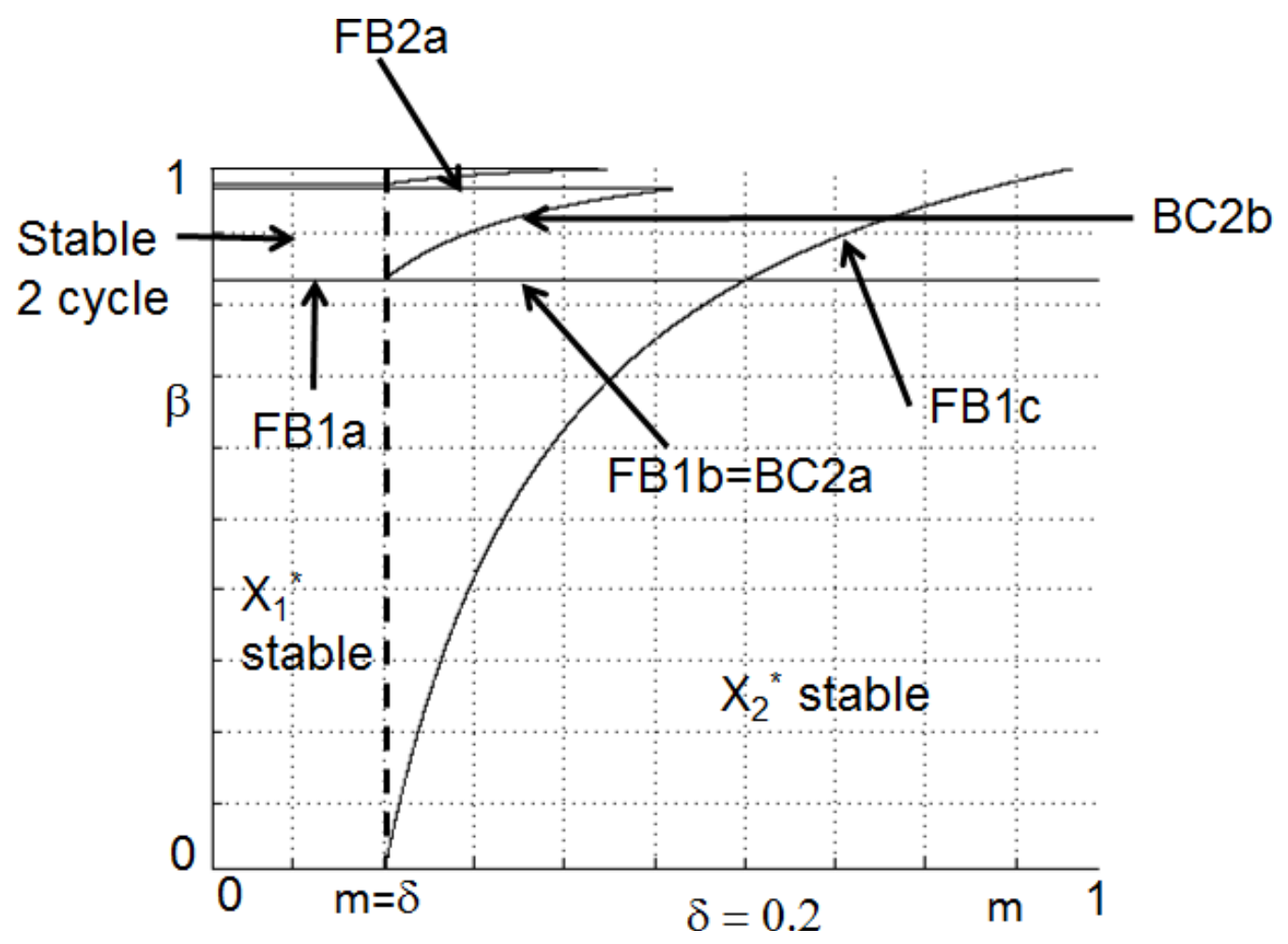

Figure 6. Map (1). In the $(m, \beta)$ plane, $\delta=0.2$, bifurcation curves of the map $\mathrm{F}$ related to fixed points and order 2 cycles.

When there exists a stable order 2 cycle, this cycle can undergo a degenerate flip bifurcation, which gives rise to an order 4 cyclic chaotic attractor. The bifurcation curve is given by the following equation, corresponding to the slope of $F^{2}$ that becomes equal to -1 :

$$
F B 2 a: \beta=\frac{1}{1+\delta^{2}}
$$

$F B 2 a$ corresponds to the slope of $F^{2}$ equal to $p_{1} p_{2}\left(F^{2}=F_{1} F_{2}\right)$. It is impossible to obtain a flip bifurcation for $F^{2}=F_{1} F_{3}$, because $p_{1} p_{3}$ can never be negative.

Other bifurcation curves correspond to border collision bifurcations. It is possible to have $F^{2}\left(x_{a}\right)=x_{a}$ or $F^{2}\left(x_{b}\right)=x_{b}$. The corresponding curves are respectively denoted $B C 2 a$ and $B C 2 b$. Their equations are given by:

$$
\begin{aligned}
& B C 2 a: \beta=\frac{1}{1+\delta} \\
& B C 2 b: \beta=\frac{m-\delta^{2}}{m\left(1-\delta^{2}\right)}, m>\delta^{2}
\end{aligned}
$$

Let us remark that $B C 2 a=F B 1 b$. Figure 5 illustrates this case. $\left(Y_{1}, Y_{2}\right)$ is an order 2 cycle. All points between $Y_{1}$ and $Y_{2}$ are points of order 2 cycles, there exist infinitely many of them. This is why the flip bifurcation is a degenerate one $(F B 1 b)$. At the same time, $Y_{2}$ is merging with $x_{b}$, this is the border collision bifurcation $(B C 2 a)$.

\subsubsection{Bifurcations of order $k$ periodic orbits}

Using the same method as for fixed points and order 2 cycles, we can obtain the bifurcation curves for order $k$ cycles. It is necessary to look at the way of exchange of points by the map $F$. The exchange depends upon the location of each point and the determination of $F, F_{1}, F_{2}$ or $F_{3}$, which has to be applied. In this paragraph, 


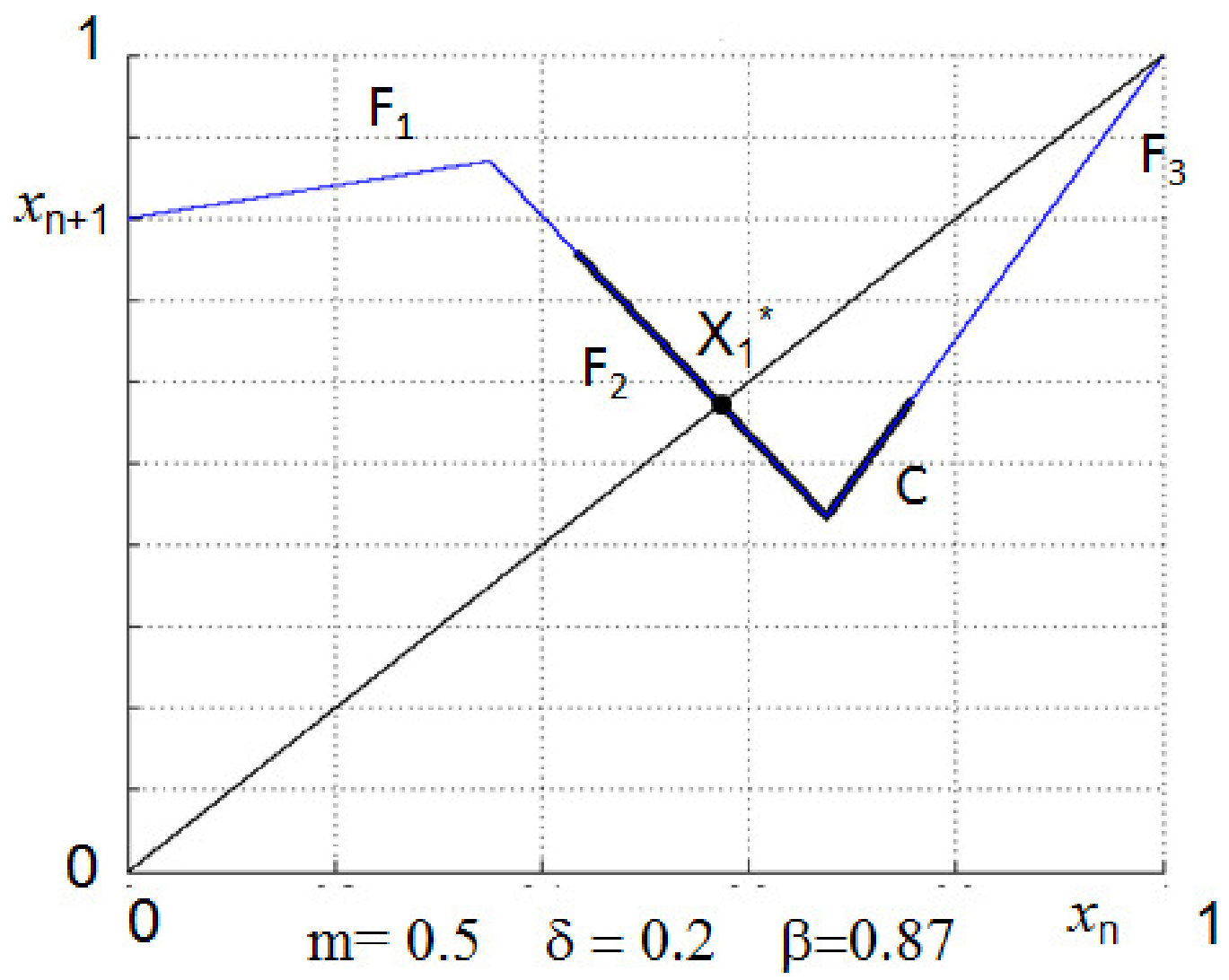

Figure 7. Map (1). An one-piece chaotic attractor $C$ appears after an homoclinic bifurcation. Parameter values are $\delta=0.2, m=0.5$ and $\beta=0.87$.

we do not intend to study and write the bifurcation curves equations for all the categories of order $k$ cycles that can be defined, but only for those that we have obtained in numerical simulations. It is clear that in simulations and for a practical use, small orders of cycles are of interest. This is why we give the analytical equations of bifurcation curves for cycles, which order is up to 7 . We have generalized when it was possible to the equation of bifurcation curves for any order. Moreover, it is worth of noting that previous studies concerning bimodal piecewise linear maps have been done in a more general way [15] [21].

First, we give the bifurcation curves corresponding to the degenerate flip bifurcations. Indeed, if we consider an order $k$ cycle, each point $X$ of this cycle verifies $F^{k}(X)=X$ and $F^{k}$ is obtained by a combination of its three determinations $F_{1}, F_{2}$ and $F_{3}$. We can write that, in $F^{k}, F_{1}$ appears $i$ times, $F_{2} j$ times and $F_{3} l$ times, with $i+j+l=k$. So regarding the degenerate flip bifurcation, it is obtained by considering the three slopes $p_{1}, p_{2}$ and $p_{3}$ such that $p_{1}^{i} p_{2}^{j} p_{3}^{l}=-1$. We have:

Proposition 1 For the map (1), the equation of the degenerate flip bifucation of an order $k$ cycle is:

$$
F B k_{i j l}: \delta^{k}=\frac{(1-\beta)^{j+l} m^{l}}{(1-\beta m)^{l} \beta^{j}}, i+j+l=k,
$$

This result is obtained, using: 


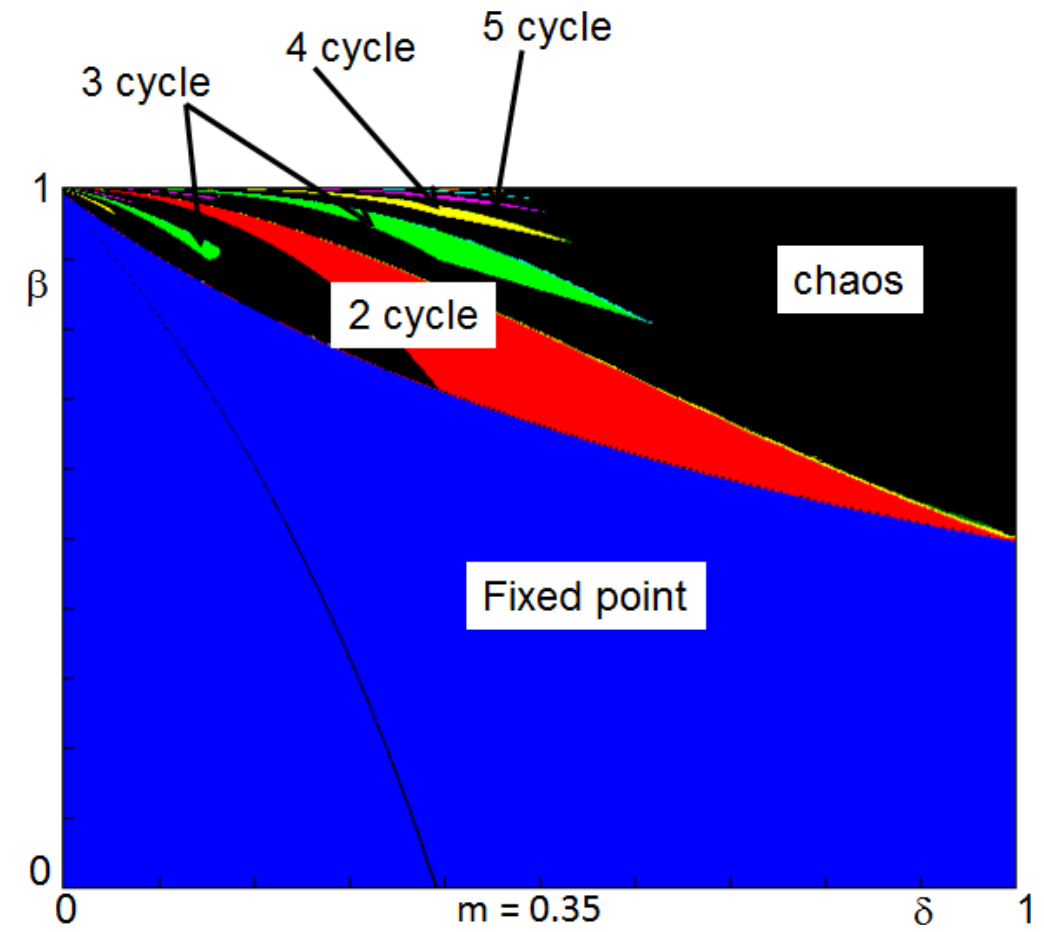

Figure 8. Map (1). Stability areas of $k$ periodic orbits, $k=1, \ldots, 5$ in the parameter plane $(\delta, \beta)$ for $m=0.35$.

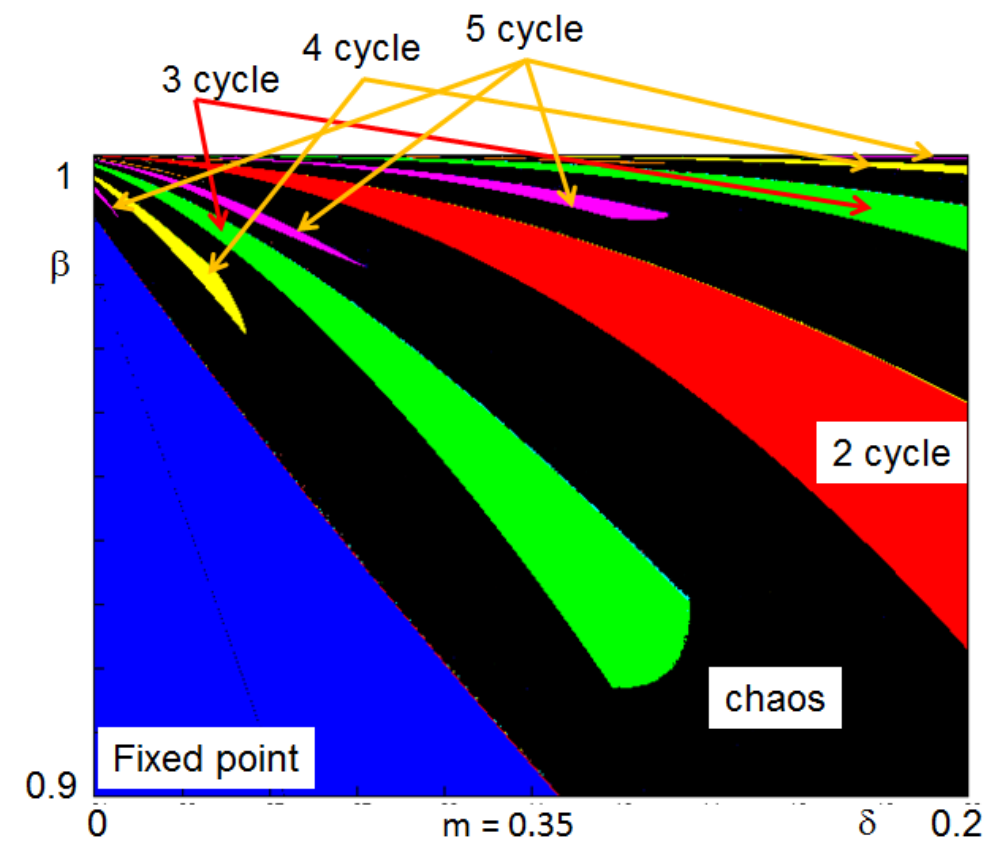

FiguRE 9. Enlargment of Fig. 8. Stability areas of $k$ periodic orbits, $k=1, \ldots, 5$ in the parameter plane $(\delta, \beta)$ for $m=0.35$. They are limited by bifurcation curves obtained in Fig. 10 . 


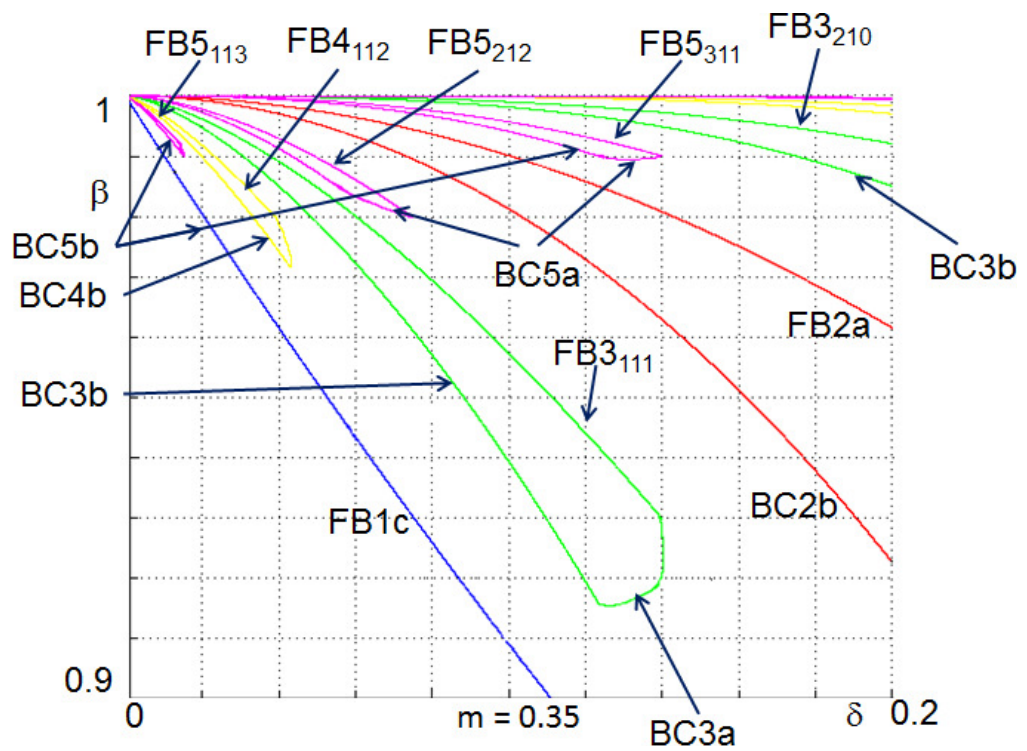

Figure 10. Map (1). Bifurcation curves of $k$ periodic orbits, $k=1,2,3,4,5$ in the parameter plane $(\delta, \beta)$ for $m=0.35$. They limit the stability areas of Fig. 9 .

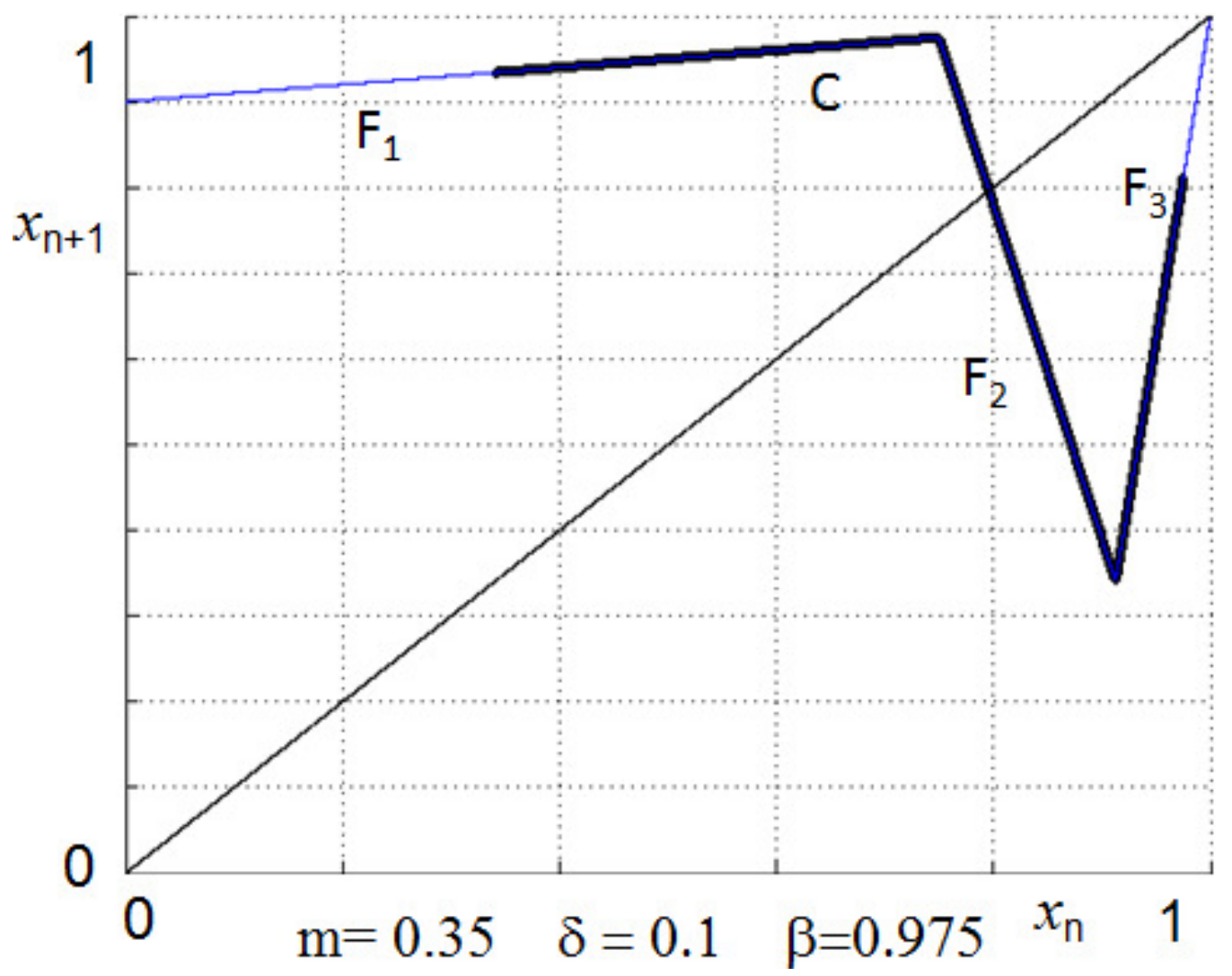

Figure 11. Map (1). A chaotic attractor is located in the three intervals $I_{1}, I_{2}$ and $I_{3}$ and is issued from an order 3-cycle whose points are exchanging using the three determinations of $F$. 


$$
p_{1}^{i} p_{2}^{j} p_{3}^{l}=\delta^{i}\left(\frac{\delta \beta}{\beta-1}\right)^{j}\left(\frac{\delta}{m} \frac{1-\beta m}{1-\beta}\right)^{l}=-1, i+j+l=k
$$

All the degenerate flip bifurcation curves are known analytically and can be plotted in the parameter planes $(\beta, \delta), m$ being fixed or $(m, \delta), \beta$ being fixed.

Border collision bifurcation curves can also be obtained. The collision can occur with any of both points $x_{a}$ or $x_{b}$. So, we have to look the parameter values for which one has $F^{k}\left(x_{a}\right)=x_{a}$ or $F^{k}\left(x_{b}\right)=x_{b}$. It is necessary to determine the way of exchanging the points of cycles by the three determinations of $F$.

The simplest case occurs when an order $k$ cycle has its points exchanged $k-1$ times by $F_{1}$ and one time by $F_{2}$ or $k-1$ times by $F_{1}$ and one time by $F_{3}$. It is possible to obtain the border collision bifurcation curves, which are denoted $B C k a$ if the collision occurs with $x_{a}$, that means $F^{k}\left(x_{a}\right)=x_{a}$, or $B C k b$ if the collision occurs with $x_{b}$, that means $F^{k}\left(x_{b}\right)=x_{b}$. We have the following result:

$$
\begin{aligned}
& B C k a: \beta=\frac{1-\delta^{k-1}}{1-\delta^{k}}, m<\delta \\
& B C k b: \beta=\frac{m-\delta^{2}}{m\left(1-\delta^{2}\right)}, m>\delta^{2}
\end{aligned}
$$

Regarding the exchange of points $k-1$ times by $F_{1}$ and one time by $F_{2}$ or $k-1$ times by $F_{1}$ and one time by $F_{3}$, we obtain a period-adding sequence of periodic orbits (see [9]) for more details. This is a classical result, which has been obtained for other maps of the same kind (see [23]).

On Fig. 8, we consider the parameter plane $(\delta, \beta), m$ being fixed and equal to 0.35 , we can see the stability areas of some order $k$ cycles, $k=1, \ldots 5$. On Fig. 9, an enlargment is given. Those cycles have their points exchanged using the three determinations of $F$. All the stability areas in Fig. 9 are bounded by degenerate flip bifurcation curves $F B k_{i j l}$ (14) or border collision bifurcation curves. The border collision bifurcation curves must be calculated by considering the exchange of the points of the cycle and the point $x_{a}$ or $x_{b}$ with which there is a collision. The cycles can be classified in different categories, regarding the way of exchanging their points. It is also possible to use Symbolic Dynamics of a bimodal map [14] [17], we have chosen to represent a cycle by beginning with the point at the left of the interval [0,1] (generally, this point is located inside $I_{1}$ and $F_{1}$ is applying). The use of symbolic dynamics corresponds to give a code to every order $k$ cycle by considering the location of the points in the intervals $I_{1}, I_{2}$ or $I_{3}$; generally, the following rule is used: a point of the cycle is coded by $L$ when it belongs to $I_{1}, M$ when it belongs to $I_{2}$ and $R$ when it belongs to $I_{3}$ (sometimes, the codes $A$ or $B$ can be used if one point of the cycle is merging with $x_{a}$ or $x_{b}$, in this paper, we will not take this possibility into account). We can propose the following classification and give the equations of the border collision bifurcation curves for some of them (let us recall that the degenerate flip bifurcation curves are given by $(14)(15))$. We obtain the following result [9]:

Proposition 2 For the map (1), the equations of the border collision bifucation of an order $k$ cycle are:

- Order $k$ cycle $\left(F_{2} F_{1}^{k-1}, L^{k-1} M\right)$, where the symbol L appears $(k-1)$ times. The equation is given by (16).

- Order $k$ cycle $\left(F_{2} F_{3}^{k-2} F_{1}, L R^{k-2} M\right)$, where the symbol $R$ appears $(k-2)$ times. The border collision bifurcation curves are given by:

$$
\begin{aligned}
& B C k a: \frac{\delta^{k}(1-\beta m)^{k-2} \beta+(1-\beta)^{k-1} m^{k-2}}{\delta(1-\beta)^{k-2} m^{k-2}}=1, \\
& B C k b: \delta^{k}=\left(\frac{(1-\beta) m}{(1-\beta m)}\right)^{k-1}
\end{aligned}
$$

- Order $k$ cycle, $k$ odd, $\left(F_{2}\left(F_{3} F_{1}\right)^{\frac{(k-1)}{2}},(L R)^{\frac{(k-1)}{2}} M\right)$, where the symbol LR appears $\left(\frac{k-1}{2}\right)$ times. The border collision bifurcation curves are given by:

$$
\begin{aligned}
& B C k a: \frac{\delta^{k-2}(1-\beta m)^{\frac{k-3}{2}}}{m^{k-4}(1-\beta)^{\frac{k-1}{2}}}-\frac{\delta^{k} \beta(1-\beta m)^{\frac{k-1}{2}}}{m^{k-3}(1-\beta)^{\frac{k+1}{2}}}=1, \\
& B C k b: \delta^{k}=\left(\frac{(1-\beta) m}{(1-\beta m)}\right)^{\frac{k+1}{2}}
\end{aligned}
$$


- Order $k$ cycle, $k$ odd, $F_{1} F_{2} F_{1}\left(F_{3} F_{1}\right)^{\frac{(k-2)}{3}},(L R)^{\frac{(k-2)}{3}} L M L$, where the symbol LR appears $\frac{k-3}{2}$ times. The border collision bifurcation curves are given by:

$$
\begin{aligned}
& B C k a: \frac{\delta^{2}}{(1-\beta)}-\frac{\delta^{k} \beta(1-\beta m)^{\frac{k-3}{2}}}{m^{\frac{k-3}{2}}(1-\beta)^{\frac{k-1}{2}}}=1, \\
& B C k b: \delta^{k}=\left(\frac{(1-\beta) m}{(1-\beta m)}\right)^{k-3}
\end{aligned}
$$

We have also observed two order 7 cycles, for which we give the border collision bifurcation curves:

- Concerning order 7 cycle $\left(F_{2}\left(F_{3}^{2} F_{1}\right)^{2}, L R R L R R M\right)$, the bifurcation curves are:

$$
\begin{aligned}
& B C k a:-\frac{\delta^{7} \beta(1-\beta m)^{4}}{m^{4}(1-\beta)^{5}}+\frac{\delta^{4}(1-\beta m)^{2}}{m^{2}(1-\beta)^{3}}=1, \\
& B C k b: \delta^{7}=\left(\frac{(1-\beta) m}{(1-\beta m)}\right)^{5}
\end{aligned}
$$

- Concerning order 7 cycle $\left(F_{2} F_{1}^{2} F_{3} F_{1}^{3}, L L L R L L M\right)$, the bifurcation curves are:

$$
\begin{aligned}
& B C k a: \frac{\delta^{3}}{(1-\beta)}-\frac{\delta^{7} \beta(1-\beta m)}{m(1-\beta)^{2}}=1, \\
& B C k b: \delta^{7}=\left(\frac{(1-\beta) m}{(1-\beta m)}\right)^{2}
\end{aligned}
$$

Bifurcation curves for order $k$ cycles, $k=1, \ldots, 7$ are plotted on Fig. 10. They clearly limit the stability areas of order $k$ cycles of Fig. 9. The curves of degenerate flip bifurcations and border collision with $x_{b}$ are given in an explicit form and can be directly plotted, the curves related to the border collision with $x_{a}$ are given under an implicit form and have to be plotted by using a numerical method (Newton-Raphson).

Between two stability regions, there exists a chaotic attractor. The chaotic attractor issued from a degenerate flip bifurcation is a cyclic one, then after a succession of homoclinic bifurcations, it becomes a one-piece chaotic one.

For some parameter values, chaos can be located on the three domains $I_{1}, I_{2}$ and $I_{3}$ (Fig. 11). This chaos is obtained from border collision bifurcations of order k-cycles whose points exchange by $F_{1}, F_{2}$ and $F_{3}$. This is the case around the stability area of the order 3-cycle $\left(F_{2} F_{3} F_{1}, L R M\right)$ (see Fig. 9 and 10). In this case, chaos can be considered as robust; indeed its existence domain in the parameter space is large enough.

A further study will be to consider the cyclic chaotic attractors and to try to limit the areas where they can exist before becomming attractors in a single piece.

\subsection{The $2 \mathrm{D}$ circuit}

Previous studies of the map (4) have been done in [4] [7] [10], they concern some specific cases. The map (4) depends upon four parameters; in [7], the study has been done for $\mu=1$ and in [4] and [10], the case of $\rho=1$ has been studied. Here, we present some results in the general case as those given in [8].

First, we have the following result:

Proposition 3 For the map (4), the four cases related to $\mu$ or $\rho$ less than or greater than 1 are topologically conjugated, whatever be $\alpha$ and $\delta$.

The proof follows immediately due to the following property:

$$
\begin{aligned}
& G_{1}\left(x_{n}, y_{n}, \alpha, \delta, \mu, \rho\right)=G_{1}\left(y_{n}, x_{n}, \alpha \rho, \delta^{1 / \mu}, 1 / \mu, 1 / \rho\right) \\
& G_{2}\left(x_{n}, y_{n}, \alpha, \delta, \mu, \rho\right)=G_{3}\left(y_{n}, x_{n}, \alpha \rho, \delta^{1 / \mu}, 1 / \mu, 1 / \rho\right)
\end{aligned}
$$

This proposition permits to reduce the study to only one case. Here we consider $\mu<1$ and $\rho<1$.

Then, let us remark that $G$ can be written under the following form: 


$$
\begin{aligned}
& G_{1}(x, y)=\left[\begin{array}{cc}
\delta & 0 \\
0 & \delta^{1 / \mu}
\end{array}\right]\left[\begin{array}{l}
x \\
y
\end{array}\right]+\left[\begin{array}{l}
\alpha-\alpha \delta \\
\alpha \rho-\alpha \rho \delta^{1 / \mu}
\end{array}\right] \\
& G_{2}(x, y)=\left[\begin{array}{cc}
\frac{\delta}{1-\alpha} & 0 \\
A(x) & \delta^{1 / \mu}
\end{array}\right]\left[\begin{array}{l}
x \\
y
\end{array}\right]+\left[\begin{array}{l}
\frac{-\alpha \delta}{1-\alpha} \\
-\alpha \rho \delta^{1 / \mu}
\end{array}\right] \\
& G_{3}(x, y)=\left[\begin{array}{ll}
\delta & B(y) \\
0 & \frac{\delta^{1 / \mu}}{1-\alpha \rho}
\end{array}\right]\left[\begin{array}{l}
x \\
y
\end{array}\right]+\left[\begin{array}{l}
-\alpha \delta \\
\frac{-\alpha \rho \delta^{1 / \mu}}{1-\alpha \rho}
\end{array}\right]
\end{aligned}
$$

From (24), we can remark that $G_{1}$ is affine with the part depending upon $x$ and $y$ that can be written from a diagonal matrix. Concerning $G_{2}$ and $G_{3}$, they also are affine, with the part depending upon $x$ and $y$ that can be written by the way of triangular matrices. So, we can deduce that $G$ is nearly linear and the dynamics and the bifurcations can mainly be obtained from the linear part. For instance, only the diagonal terms play an important role to detect bifurcations of periodic orbits.

As in the case of the 1D circuit, we can obtain analytically some bifurcation curves related to the case where two determinations of the map have to be considered to obtain the attractors. We have the following proposition:

Proposition 4 For the map (4), equations of the degenerate flip bifurcation curves related to $G^{k}=G_{1}^{k-1} G_{2}$ or $G^{k}=G_{1}^{k-1} G_{3}$ are:

$$
\begin{aligned}
& \text { DFBka: } \delta=(\alpha-1)^{\frac{1}{k}} \text { for } G_{1}^{k-1} G_{2} \\
& D F B k b: \delta=(\alpha \rho-1)^{\frac{\mu}{k}} \text { for } G_{1}^{k-1} G_{3}
\end{aligned}
$$

These curves [7] [10] are related to exchange of points using $G_{1}$ and $G_{2}$ (DFBka) or $G_{1}$ and $G_{3}$ (DFBkb) and are obtained by considering one eigenvalue of the Jacobian of $G_{1}^{k-1} G_{2}$ or $G_{1}^{k-1} G_{3}$ equal to -1. Figure 12 illustrates the case of $D F B 1 a$, a fixed point undergoes a flip bifurcation and there exist a curve of infinitely many order 2 cycles at the bifurcation. We also want to focus on the possibility of exchange of points using the three determinations $G_{1}, G_{2}$ and $G_{3}$ of the map. We can obtain the curves BCBk (Figure 13), which correspond to border collision bifurcations, in a particular case when considering the k-cycles ( $k$ even) obtained using:

$$
G_{1}^{\frac{k}{2}-1} G_{3} G_{1}^{\frac{k}{2}-1} G_{2}
$$

These curves are obtained numerically, they correspond to a point of the k-cycle ( $k$ even) merging with the border $x=1$. After the border collision bifurcation, a chaotic attractor exists, which is located in the three domains $D_{1}, D_{2}$ and $D_{3}$ (Figure 16). These curves also correspond to limit of stability areas of periodic attractors (Figure 14). Figure 15 correspond to a bifurcation diagram with $\alpha, \rho$ and $\mu$ fixed $(\mu=0.29$, see Figures 13 and 14 where the line $\mu=0.29$ is given), we can see the succession of periodic orbits (order $k=1,2,4,3,6,4,8$ ) and chaotic attractors between two periodic windows and after the existence of periodic windows.

Our circuits have also been implemented and the existence of periodic or chaotic attractors have been put in evidence in the implementations in spite of noise inherent to such experimentations. Figures 17 and 18 show an order 3 cyclic chaotic attractor respectively obtained experimentally and by Matlab simulations.

\section{CONCLUSion}

We have proposed two examples of simple circuits permitting to obtain chaotic signals. Both circuits are modeled using maps with three determinations in the phase space. For both models, it is possible to obtain analytically the equations of some bifurcation curves. It permits to understand the appearance of chaos by border collision bifurcations. Such chaos can be robust. Chaos can also be obtained using the three possible 


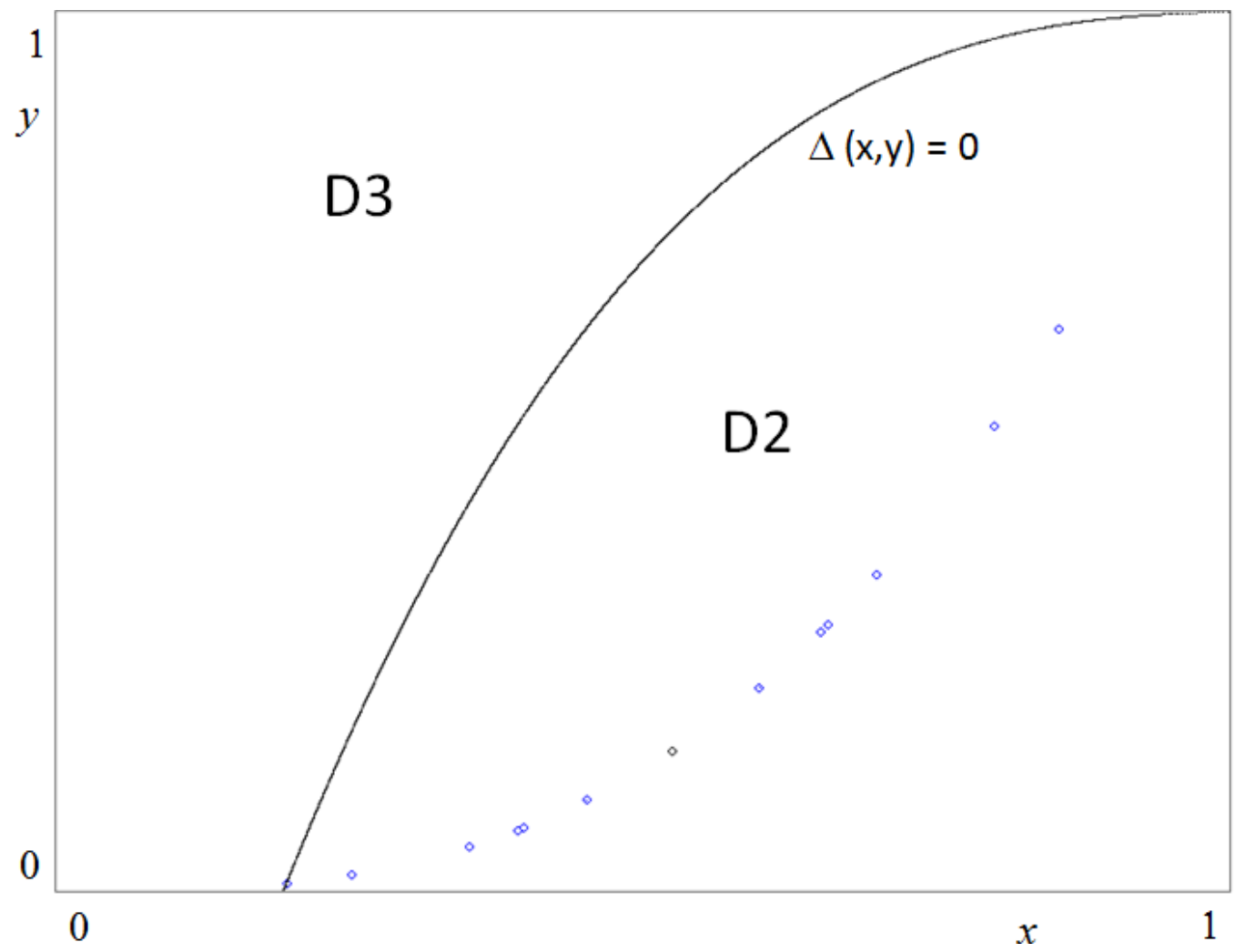

FigURE 12. For the map (4), in the phase space $(x, y)$, degenerate flip bifurcation (DFB1a) for $\alpha=1.053, \mu=0.35, \delta=0.053$ and $\rho=0.95$. A curve of infinitely many order 2 cycles exist at the bifurcation. Some of them are plotted on the figure.

determinations, but as the parameter values that permit to obtain it are in smaller domains of parameter space, such chaos does not seem so robust in our examples. Nevertheless, chaos obtained using the three determinations of the map could be more useful for applications (for instance, in secure communications, it could be harder to detect it).

We intend to continue and develop our studies in order to improve the way of obtaining more robust chaos useful for applications. For instance, depending on parameter values, chaos can appear as cyclic chaotic attractor or as a single piece chaotic attractor. Of course, with the aim of using it in applications, it seems better to consider a single piece attractor instead of a cyclic one with different pieces. So, it can be of interest to limit such areas in parameter space. In this paper, we have considered deterministic chaos. From another point of view, statistical studies of our chaotic signals could be of interest. Indeed, chaotic signals can be used for applications, for instance cryptography or secure transmissions and it is important to evaluate their randomness. Then, it can be of great interest to apply the statistical NIST tests [20] or to study the auto-correlation and cross-correlation of such signals.

\section{REFERENCES}

[1] S. Banerjee, J.A. Yorke, C. Grebogi, "Robust chaos", Physical Review Letters, 80, pp. 3049-3052, 1998.

[2] S. Banerjee, P. Ranjan, C. Grebogi, "Bifurcations in 2D piecewise smooth maps - theory and applications in switching circuits", IEEE Trans. Circuits Systems I, 47, pp. 633-643, 2000.

[3] E. Blokhina, J. Pons, J. Ricart, O. Feely, M. Pumar, "Control of MEMS vibration modes with pulsed digital oscillators", IEEE Trans. Circuits Systems I, 57 (8) (2010) 1865-1878. 


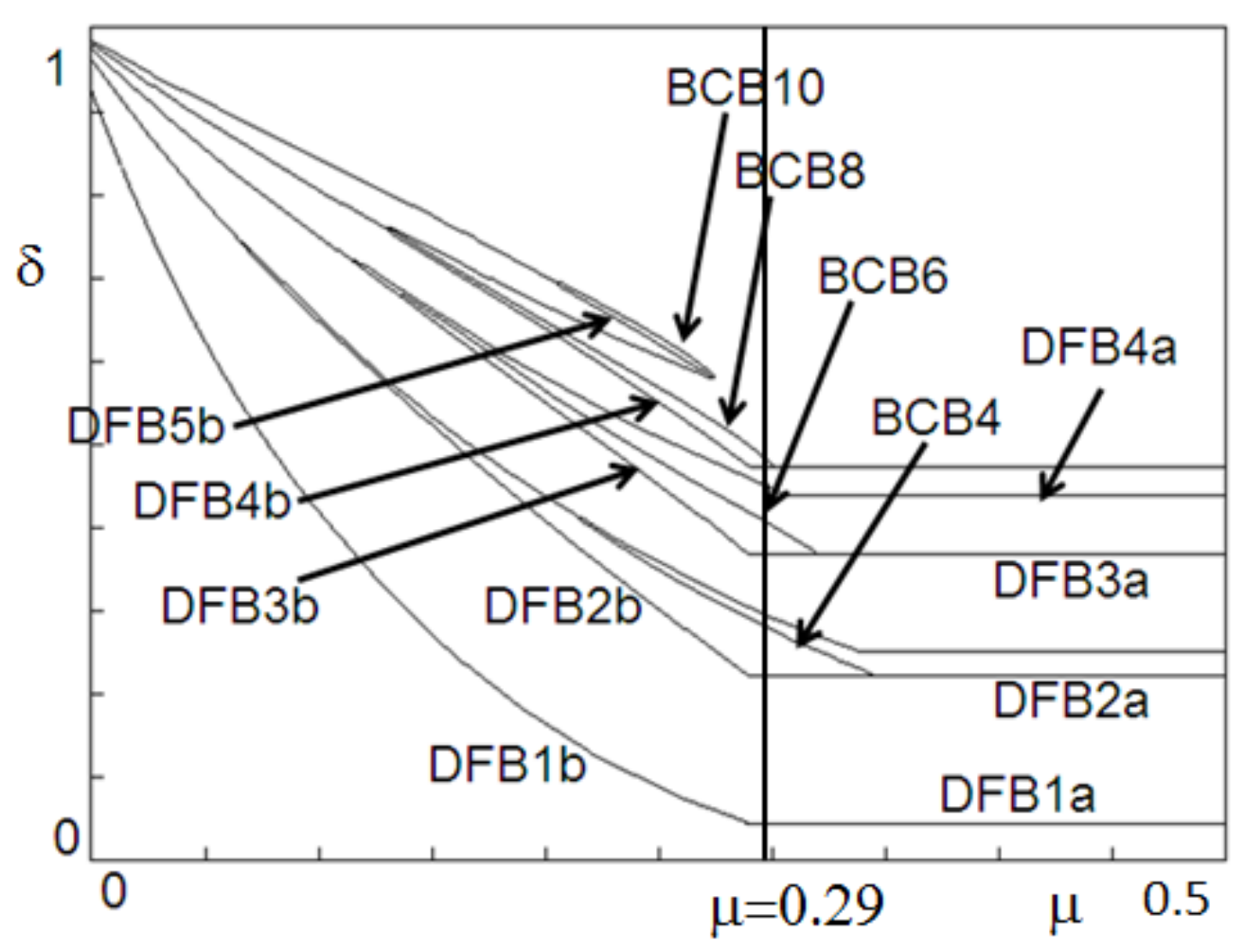

FiguRE 13. Some bifurcation curves for the map (4) in the parameter plane $(\mu, \delta)$ when $\alpha=$ 1.053 and $\rho=0.95$.

[4] P. Chargé, D. Fournier-Prunaret, L. Gardini, "Two-dimensional Piecewise defined Maps realized with a Simple Switching Circuit", International Conference on Nonlinear Theory and Applications (NOLTA'08), Proceedings, Budapest, Hungary, September 2008.

[5] R.L. Devaney, "An introduction to chaotic dynamical systems", Addison Wesley Publishing Company New York, 1989,181 p.

[6] D. Fournier-Prunaret, P. Chargé, "Bifurcation Structure in a Circuit Modeled by a 1-Dimensional Piecewise Linear Map", IEICE International Conference on NOnLinear Theory and Applications (NOLTA'11), Proceedings, Kobe, Japan, September 2011.

[7] D. Fournier-Prunaret, P. Chargé, L. Gardini, "Border Collision Bifurcations and Chaotic Sets in a Two-dimensional Piecewise Linear Map", Communications in Nonlinear Science and Numerical Simulations, Vol. 16, n 2, pp. 916-927, 2011.

[8] D. Fournier-Prunaret, P. Chargé, L. Gardini, "Chaos generation from 1D or 2D circuits including switches", IEEE International Conference for Internet Technology and Secured Transactions (ICITST), Dubaï, UAE, December 12-13, pp. 86-90, 2011.

[9] D. Fournier-Prunaret, P. Chargé, "Route to chaos in a circuit modeled by a 1- dimensional piecewise linear map", IEICE Nonlinear Theory and its Applications, Vol.3, (4), pp. 521-532, October 2012, 12427.

[10] L. Gardini, D. Fournier-Prunaret, P. Chargé, "Border Collision Bifurcations in a Two-Dimensional PWS Map from a Simple Circuit", Chaos 21, 023106 (2011), doi:10.1063/1.3555834.

[11] Hayashi, C., Y. Ueda and H. Kawakami, "Solution of Duffing's equation using mapping concepts", Proc. 4th Int. Conf. Nonlinear Oscillations Prague, pp. 25-40 (1968).

[12] T. Kousaka, T. Kido, T. Ueta, H. Kawakami, M. Abe, "Analysis of Border-Collision Bifurcation in a Simple Circuit", IEEE International Symposium on Circuits and Systems (ISCAS'00), Geneva, Switzerland, May 2000.

[13] T. Kousaka, Y. Yasuhara, T. Ueta, H. Kawakami, "Experimental Realization of Controlling Chaos in the Periodically Switched Nonlinear Circuit", Int. J. Bifurcation and Chaos, Vol. 14, (10), pp. 3655-3660, 2004.

[14] J.P. Lampreia, J. Sousa Ramos, "Symbolic dynamics of bimodal maps", Portugaliae Mathematica, vol. 54, n¹, pp. 1-18, 1997.

[15] Yu. L. Maistrenko, V.L. Maistrenko, S.I. Vikul, "On period-adding sequences of attracting cycles in piecewise linear maps", Chaos, Solitons and fractals, vol. 9, (1/2), pp. 67-75, 1998. 


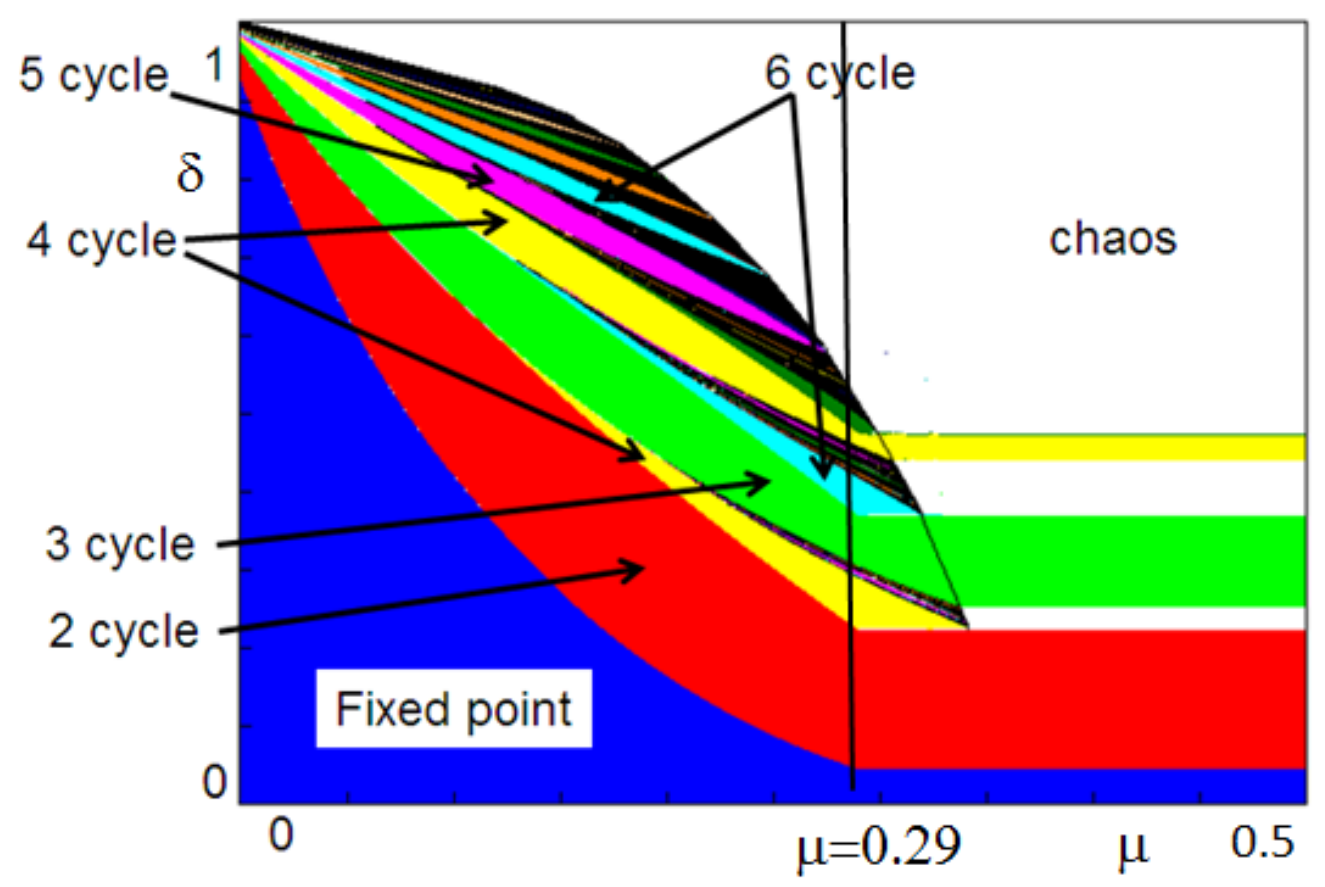

FiguRe 14. Stability areas for the map (4) in the parameter plane $(\mu, \delta)$ when $\alpha=1.053$ and $\rho=0.95$. The areas are limited by the bifurcation curves of the Figure 13 . The white area corresponds to the existence of a robust chaotic attractor located in the $D_{1}$ and $D_{2}$ domains, whereas the black parts correspond to the existence of a chaotic attractor located in the three domains $D_{1}, D_{2}$ and $D_{3}$. In this last case, chaos can be less robust, because its domain of existence in the parameter space is small.

[16] S. Mandal, S. Banerjee, "An Integrated CMOS Chaos Generator", National Conference on Nonlinear and Dynamics, Indian Institute of Technology, December 2003.

[17] N. Martins, R. Severino, J. Sousa Ramos, "Isentropic Real Cubic Maps", Int. J. of Bifurcation and Chaos in Applied Sciences and Engineering, vol. 13, (7), pp. 1701-1709, 2003.

[18] T. Matsumoto, L. 0. Chua and M. Komuro, "The double scroll", IEEE Trans. Circuits Syst., vol. CAS-32, pp. 797-818, Aug. 1985.

[19] G. Mazzini, G. Setti, R. Rovatti, "Chaotic complex spreading sequences for asynchronous DS-CDMA. I. System modeling and results", IEEE Trans. Circuits Systems I, 44 (10) (1997) 937-947.

[20] NIST (National Institute of Standards and Technology) tests, http://csrc.nist.gov/groups/ST/toolkit/rng/index.html.

[21] A. Panchuk, "Three segmented piecewise linear map", International Workshop on Nonlinear Maps and Applications (NOMA'11), Evora, Portugal, September 15-16, 2011.

[22] R. Clark Robinson, "An introduction to dynamical systems: discrete and continuous", American mathematical Society, 2012, vol. 19, 733 p, ISBN-10: 0-8218-9135-9.

[23] I. Sushko, L. Gardini, "Degenerate Bifurcations and Border Collisions in Piecewise Smooth 1D and 2D Maps", Int. J. Bifurcation and Chaos, Vol.20, (7), pp. 2045-2070, 2010.

[24] C. Vladeanu, S. El Assad, J.C. Carlach, R. Quéré, "Improved Frey Chaotic Digital Encoder for Trellis-Coded Modulation", IEEE Trans. Circuits Systems II, 56 (6) (2009) 509-513. 
ESAIM: PROCEEDINGS AND SURVEYS

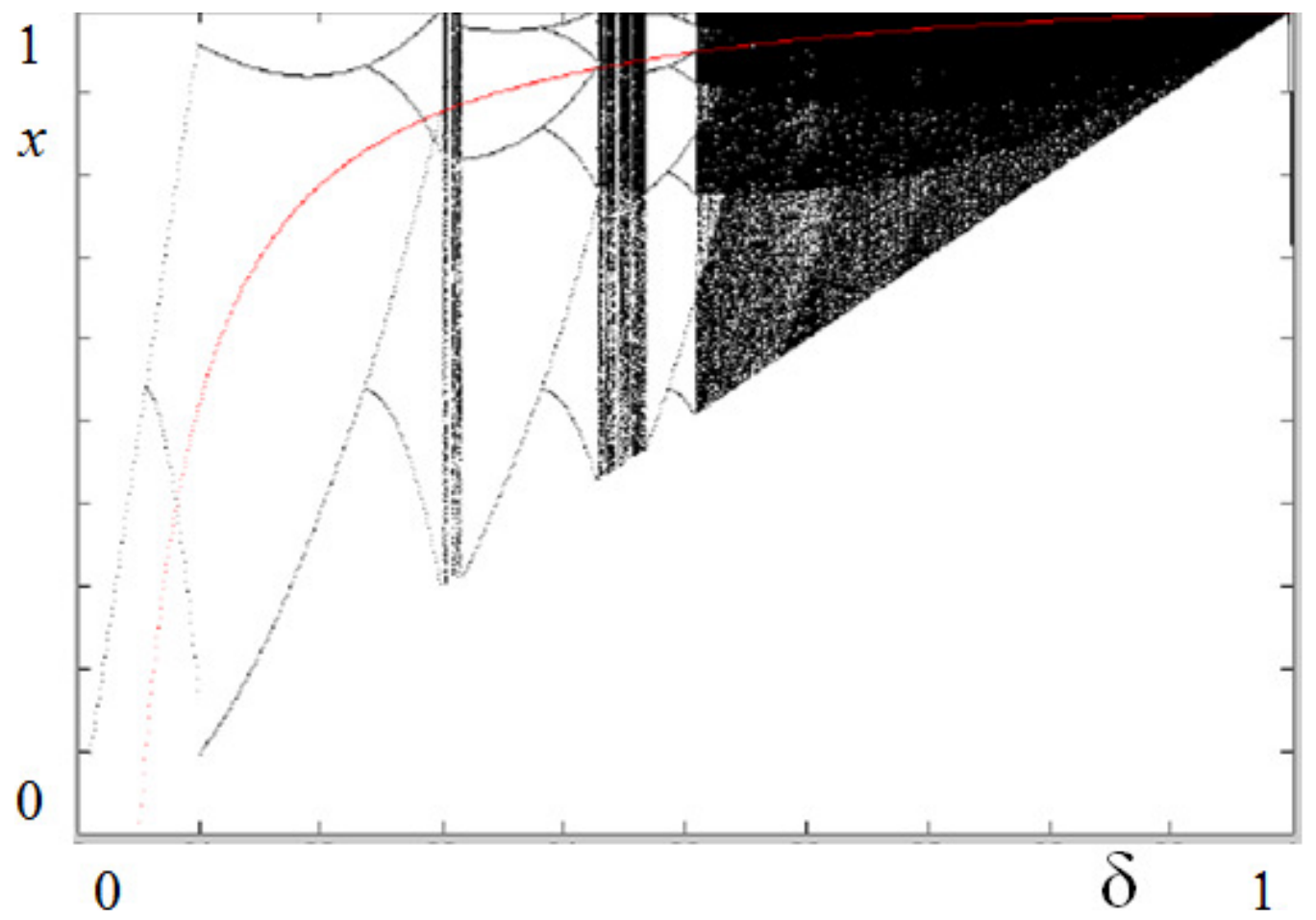

FiguRE 15. Bifurcation diagram for the map (4) in the parameter plane $(\delta, x)$ when $\mu=0.29$, $\alpha=1.053$ and $\rho=0.95$. 


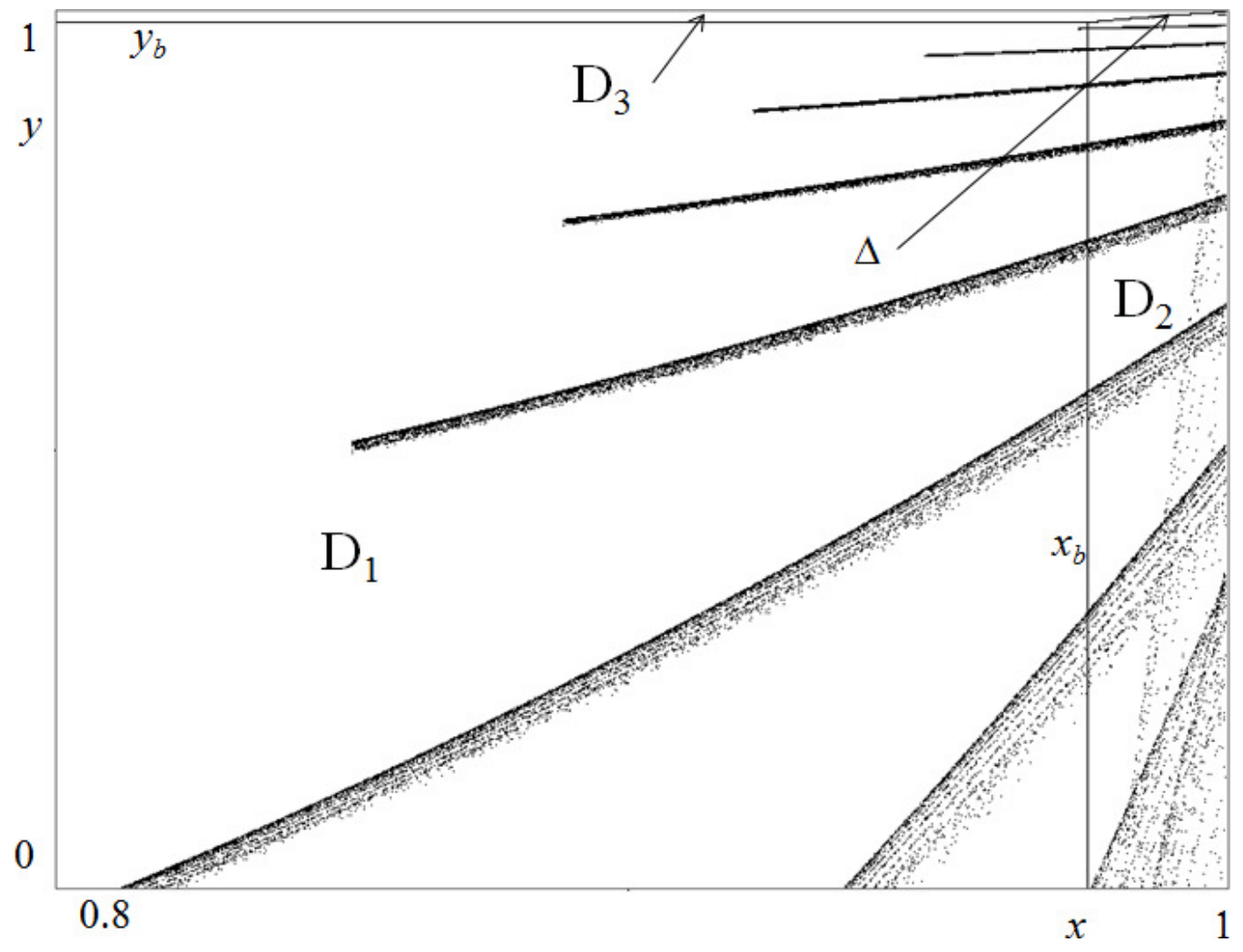

Figure 16. Chaotic attractor in the phase space $(x, y)$ for the map (4) when $\alpha=1.053$, $\mu=0.15, \delta=0.9$ and $\rho=0.95$. The attractor is located in the three domains $D_{1}, D_{2}$ and $D_{3}$.
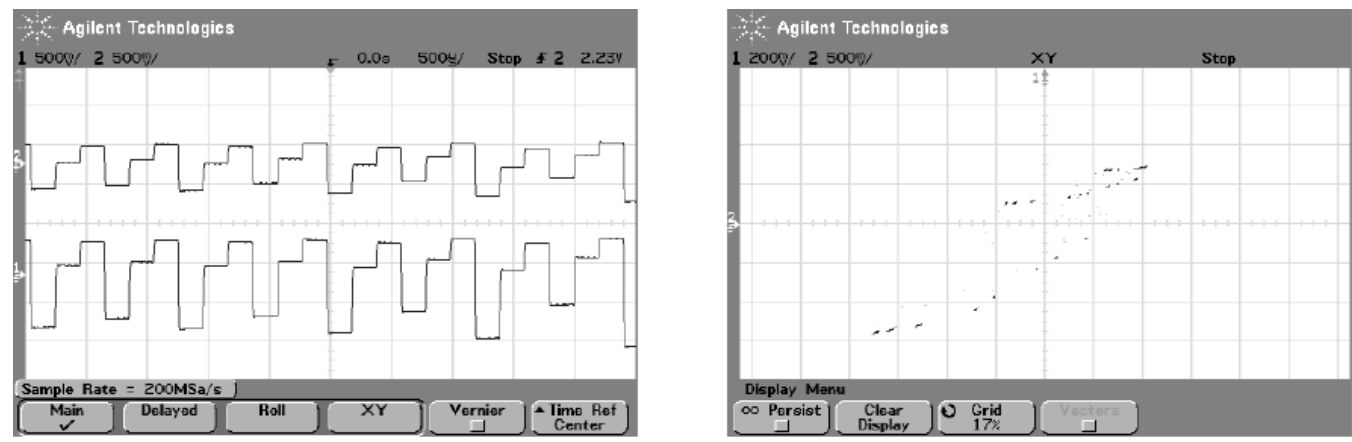

FiguRe 17. Experimental implementation of the circuit modeled by the map $G$. An order 3 cyclic chaotic attractor has been obtained for $\alpha=1.053, \mu=0.48, \delta=0.4$ and $\rho=0.95$. 


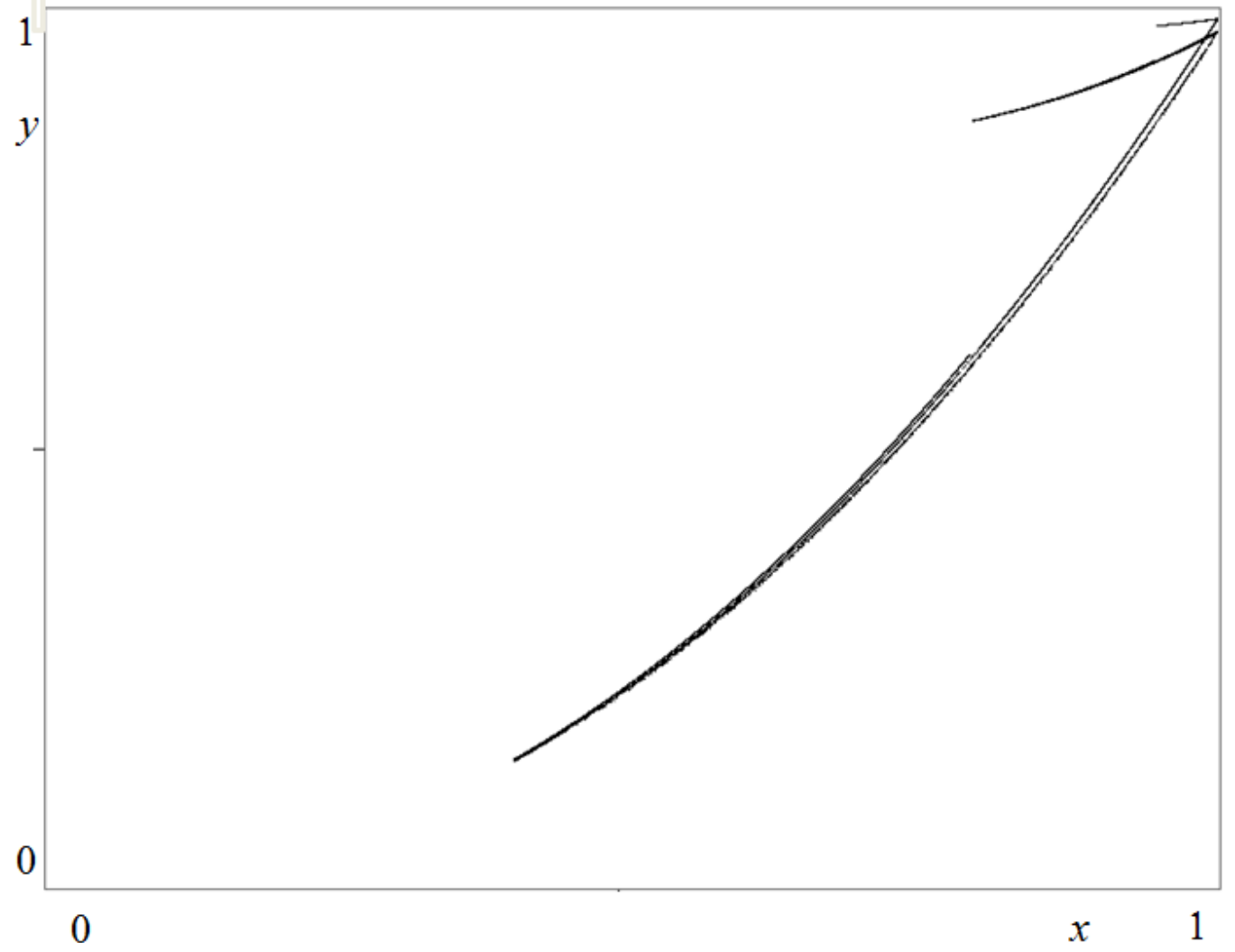

FiguRE 18. Chaotic attractor for the map (4) obtained by simulations when $\alpha=1.053, \mu=$ $0.48, \delta=0.4$ and $\rho=0.95$. It corresponds to the attractor experimentally obtained in Figure 17 . 